\section{Michael Eble}

\section{Medienmarken im Social Web}

Wettbewerbsstrategien und

Leistungsindikatoren von Online-Medien aus medienökonomischer Perspektive

Münster: Lit, 2013. - 306 S.

(Reihe Bonner Beiträge zur Onlineforschung; 5)

ISBN 978-3-643-12110-3

(Zugl.: Bonn, Univ., Diss., 2012)

„Die vorliegende Arbeit bietet als Momentaufnahme in einem hoch dynamischen Feld unter konzeptionellen Gesichtspunkten für OnlineMedien im Social Web ein Modell und einen Ansatz zu dessen Umsetzung sowie in empirischer Hinsicht einen Referenzpunkt für Wettbewerbsstrategien und Leistungsindikatoren", so der Autor in einer abschließenden Einordnung seiner Publikation. Dies macht ein Grundproblem der Arbeit deutlich: In einem immer noch und zur Zeit der Untersuchung allemal stark variablen Umfeld soll mit einer einzigen Momentaufnahme ein Modell von allgemeiner Aussagekraft und strategischer Relevanz abgeleitet werden. Das funktioniert natürlich nicht. Auch der Anspruch, für nachrichten- wie unterhaltungsdominierte Medienmarken gleichermaßen die Bedeutung von SocialMedia-Aktivitäten auf allen wichtigen Plattformen theoretisch zu fassen und erschöpfend auszumessen, ist nicht einlösbar. Gleichwohl ist dem Autor ein wichtiger und auch aufgrund der hervorragenden grafischen Darstellungen sehr gut lesbarer und lesenswerter Beitrag gelungen.

Da die Publikation ohne Vorwort direkt in die Ausgangslage und Forschungsfragen einsteigt, muss die Entstehungsgeschichte aus etwas verteilten Informationen rekonstruiert werden: Grundlage ist eine Promotionsarbeit, die zwischen Oktober 2008 und Juni 2011 entstand und an der Universität Bonn als Dissertation angenommen wurde. 2013 schließlich erfolgte die Publikation als fünfter Band der von Caja Thimm herausgegebenen Bonner Beiträge zur Onlineforschung. Die Datenerhebung bezieht sich dabei teilweise noch auf das vierte Quartal 2009, rangiert aber überwiegend über das Jahr 2010. Das ist ein Zeitraum, in dem die Social-Media-Aktivitäten der traditionellen Medienunternehmen noch stark experimentell waren. Überdies waren sich auch die werbungtreibenden Unternehmen und deren Marketing-Dienstleister noch nicht im Klaren, welche Kennzahlen der Social-Media-Plattformen überhaupt marketingrelevant und wie diese im
Gesamtportfolio mit den Leistungswerten traditioneller Werbemedien zu beurteilen wären.

Die Arbeit fällt also in eine Zeit, in der einerseits schon deutlich war, dass mit dem Social Web notwendig Veränderungen in der Markenführung einhergehen, aber noch nicht, in welche Richtungen, mit welcher Vehemenz und wie sich diese Veränderungen in die bereits seit Längerem laufende generelle Entwicklung digitaler Medienangebote und deren Verbreitung über immer neue Endgeräte einbetten lassen. Das muss man fairerweise berücksichtigen, wenn heute, fast vier Jahre nach dem empirischen Teil der Promotionsarbeit, rezensiert wird. Ein für damalige Verhältnisse hervorragender Untersuchungsteil mit zahlreichen neuen und in Teilen auch wegweisenden Einzelerkenntnissen steht ein etwas löchriges theoretisch-konzeptionelles Fundament gegenüber, an das der Autor sich bemüht anzuknüpfen. Letzteres ist nur begrenzt erfolgreich, da der im Kontext traditioneller Medien und früher Online-Angebote entwickelte Fundus nicht ausreicht und der Autor selbst seinen Fokus mehr auf explorative Empirie als auf die Theorieentwicklung legt.

Auf insgesamt 306 Seiten - darunter 21 Seiten Literaturangaben und ein 31-seitiger, sehr gut dokumentierter Anhang zu Details der Methodik, Übersichten zu einzelnen Leistungswerten sowie einer Veranschaulichung der untersuchten Social-Media-Angebote - nähert sich der Autor zunächst über drei etwa gleichgewichtige überwiegend theoretische Kapitel dem empirischen Schwerpunkt der Arbeit. Die drei rahmensetzenden Kapitel versuchen zunächst Online-Kommunikation innerhalb des Gesamtkontextes öffentlicher Kommunikation zu verorten, dann die wesentlichen Plattformen des Social Web anhand der persönlichen wie professionellen Verwertungszusammenhänge sowie der publizistischen Strukturen und Prozesse zu systematisieren, um schließlich die Führung und Bewertung von Medienmarken zu elaborieren. Dabei geht der Autor sehr gründlich auf die entsprechende kommunikationswissenschaftliche Literatur ein. Wirtschaftswissenschaftliche Überlegungen zur Bedeutung von Marken in Zeiten digitaler Medien im Allgemeinen sowie von Social Media im Besonderen kommen dabei leider etwas zu kurz. Auch macht der Autor weitgehend an der deutschen Sprachgrenze halt. Sehr gut gelungen ist die Ableitung dessen, was aus der kommunikationswissenschaftlichen Forschung zum Verstehen und Erklären von Online-Medien wohl weiterhin gültig bleibt und Anknüpfungspunkt sein kann. Weniger stark ist das Herausarbeiten 
der Schwächen und der Notwendigkeit neuer Konzepte und theoretischer Fundierung.

Darunter leidet auch das erste Teilziel, mittels Sekundäranalysen theoretische Grundlagen von Wettbewerbsstrategien sowie der Leistungsbewertung von Medienmarken zu erarbeiten. Dazu wäre es auch notwendig gewesen, Markenidentität und -images traditioneller $\mathrm{Me}-$ dienmarken in ihren verschiedenen digitalen Ablegern zu differenzieren. Weder Markenidentität und -image noch die Inhalte, geschweige denn die Gratifikationen sind deckungsgleich, und für die crossmediale Kombination der Angebote werden ganz unterschiedliche Markenarchitekturen versucht. Die Leistungswerte je Angebot müssen daher auch gekoppelt betrachtet werden. So hängt etwa der Erlös aus Bannerwerbung einerseits mit dem Setzen von Paywalls und andererseits dem diesbezüglichen Erlös durch Paid Content zusammen. Weiterhin nicht thematisiert wird die Rolle von Suchmaschinen, insbesondere Google, für die Reichweite digitaler Angebote traditioneller Medienmarken im Web. Die Suchmaschinenoptimierung (SEO) spielte schon damals eine große Rolle im redaktionellen Alltag der Online-Ableger, insbesondere der großen Nachrichtenmedien, und umfasst heute auch das Social Web (Social SEO). Überdies wirkt das Antizipieren der Reaktionen im (Social) Web in Zeiten ökonomischen Drucks auch auf die redaktionelle Arbeit zurück. Anschlusskommunikation ist daher nicht nur eine Folge der Publikation, sondern es ist davon auszugehen, dass deren Einschätzung auch schon auf das Angebot selbst Einfluss nimmt. Auch die Strategien neuer Akteure bleiben unberücksichtigt. Die gab es zum Zeitpunkt der Analyse freilich noch nicht in wettbewerbsrelevantem Umfang, gleichwohl wäre es eine wichtige Erweiterung der Perspektive gewesen zu eruieren, mit welchen Geschäftsmodellen sie den Traditionsmarken Paroli bieten wollen bzw. könnten. Dann wären auch neue Geschäftsmodelle für Medienunternehmen in Zeiten von Social Media in den Blick gerückt oder zumindest auch heute schon (ökonomisch) erfolgreich angegangene Erweiterungen wie etwa „Media for equity" oder eCommerce-Aktivitäten.

Das zweite Teilziel der Arbeit bestand im Messen und Vergleichen von Leistungswerten der Medienmarken im Social Web. Dies wird mittels der Verknüpfung von Online-Inhalts-, Nutzerdaten- und Netzwerkanalyse für verschiedene Plattformen hervorragend erreicht. Vier thematisch und methodisch unterschiedliche, aber aufeinander abgestimmte Primäruntersuchungen der Aktivitäten um traditionelle
Medienmarken im Social Web führte der Autor dazu durch: 1) Zur Anschlusskommunikation auf YouTube, 2) zu den Medienmarken ProSieben und TV Total, 3) zu den Medienmarken Wall Street Journal und New York Times sowie 4) zu den Medienmarken Spiegel Online und Focus Online. Die Arbeiten liefern einen enormen Fundus an Anhaltspunkten, welche Leistungswerte von wettbewerbsstrategischer Bedeutung für Medienunternehmen sein könnten. Sowohl in der Datenerhebung als auch in der Datenanalyse werden dazu neue Wege beschritten, an die nachfolgende Untersuchungen sehr gut anknüpfen können.

Eine Weiterführung der Untersuchungsstränge ist allerdings auch notwendig, denn beim dritten und letzten selbst gesetzten Teilziel des Autors, die Leistungswerte und Unterschiede einzuordnen, um das Modell zu reflektieren, bleiben einige Fragen offen. Das liegt auch daran, dass der Autor, der heute am Fraunhofer-Institut IAIS zuständig ist für IKT-Beratung und Business Development, die Praxisrelevanz der Arbeit auf nicht einmal einer ganzen Buchseite abhandelt. Obwohl er doch selbst mit Bezug auf Maletzke formuliert, Medienpraktiker würden durch Medienforscher enttäuscht, wenn sie von der Kommunikationsforschung Ratschläge oder Faktenwissen erwarten. An Fakten mangelt es dem Beitrag nicht. Die Handlungsrelevanz für Medienunternehmer ist leider überschaubar.

Castulus Kolo

\section{Linda Erker / Klaus Kienesberger / Erich Vogl / Fritz Hausjell (Hrsg.) \\ Gedächtnis-Verlust?}

Geschichtsvermittlung und -didaktik in der Mediengesellschaft

Köln: Halem, 2013. - 260 S.

(Reihe Öffentlichkeit und Geschichte; 6)

ISBN 978-3-86962-066-4

Der Sammelband „Gedächtnis-Verlust. Geschichtsvermittlung und -didaktik in der Mediengesellschaft" beschäftigt sich mit der Frage, wie die Geschichte von Holocaust und Nationalsozialismus in unserer heutigen Gesellschaft vermittelt werden soll. Das Thema ist von aktuellem Interesse: Angesichts des anstehenden Generationenwechsels und dem damit drohenden Erlöschen des „kommunikativen Gedächtnisses “ (Assmann \& Assmann, 1994) ist die Frage, wie eine kritische Auseinandersetzung mit diesem historischen Abschnitt in Zukunft aussehen kann. Der Sammelband verfolgt eine de- 